\title{
The fermion determinant and the chiral gauge theory on a lattice
}

\author{
Sergei V. Zenkin ${ }^{\mathrm{a}}$

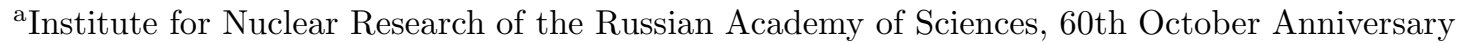 \\ Prospect 7a, 117312 Moscow, Russia
}

\begin{abstract}
Considering as an example a simple lattice ansatz for the chiral fermion determinant, we demonstrate that even very mild violation of gauge invariance by the determinant at finite lattice spacing leads to the need for another scale in the full gauge theory. This new scale is much grater than the lattice spacing and is associated with the gauge variables.
\end{abstract}

1. The aim of this paper is to examine consequences of a mild violation of gauge invariance by the chiral fermion determinant for the full gauge theory. To this end we employ a simple ansatz for the chiral fermion determinant [1] where the violation of the gauge invariance is in fact minimized. Namely, our ansatz ensures exact gauge invariance of the real part of the determinant at finite lattice spacing, in the continuum limit for smooth external fields reproduces the consistent anomalies, and for anomaly-free theories defines gauge invariant imaginary part of the determinant. The violation of the gauge invariance occurs only in the imaginary part of the determinant at finite lattice spacing and, thus, is very mild . We, however, shall demonstrate that even such a mild imperfection of the determinant leads to strong violation of the gauge invariance of the full gauge theory, where the gauge variables become dynamical, and that to suppress such a violation one can allow non-smoothness of the gauge fields only on a scale which is much grater than the lattice spacing.

We should note that the necessity for the additional scale in the context of the chiral gauge theories was discussed earlier, see, for example, [4.5], and [6] for a review and more complete references. Our consideration yields additional arguments for that.

2. Our ansatz is a lattice transcription of the

\footnotetext{
${ }^{1}$ To our knowledge among other proposals only the overlap formula 2f and also constructed ad hoc 'hybrid' formulations [3] possess such properties.
}

effective action

$$
\Gamma[A] \equiv \ln Z[A]=\operatorname{Tr} \ln \left[\partial(A) \partial^{-1}(0)\right],
$$

where $\partial(A)$ is a chiral Dirac operator, say, $\partial(A)=$ $\gamma_{\mu}\left(\partial_{\mu}+i g A_{\mu} P_{L}\right)$, where $P_{L}=\left(1+\gamma_{5}\right) / 2$. The ansatz is based on the observation that both the gauge invariance of the real part of $\Gamma[A]$, $\Re \Gamma[A]$, and the noninvariance of the imaginary part of $\Gamma[A], \Im \Gamma[A]$, can be maintained on a lattice if in the naive lattice formulation of (1) the domain of integration over fermion loop momenta in all diagrams is narrowed down to $\mathcal{D}=$ $(-\pi /(2 a), \pi /(2 a))^{D}$, i.e. to the $1 / 2^{D}$-th part of the fermion Brillouin zone $\mathcal{B}=(-\pi / a, \pi / a)^{D}$, where $a$ is the lattice spacing. In this case the fermion modes which lead to the species doubling and render any theory vector-like are no longer dangerous, for they almost decouple from smooth gauge fields, though are still important for restoring the gauge invariance of $\Re \Gamma[A]$.

Our ansatz realizes such a procedure, and reads as follows:

$\Gamma[A]=\operatorname{Tr} \Theta \ln \left[\nabla(U) \nabla^{-1}(1)\right]$,

where $\nabla(U)$ is the naive lattice transcription of the Dirac operator

$$
\begin{aligned}
\nabla_{m n}(U) & =-\sum_{\mu} \gamma_{\mu} \frac{1}{2 a}\left(U_{m m+\hat{\mu}} \delta_{m+\hat{\mu} n}\right. \\
& \left.-U_{m} m-\hat{\mu} \delta_{m-\hat{\mu} n}\right), \\
U_{m m \pm \hat{\mu}} & =\exp \left[ \pm i g a A_{\mu}(m \pm \hat{\mu} / 2) P_{L}\right],
\end{aligned}
$$

and $\Theta$ is the projection operator that cuts out the proper $1 / 2^{D}$-th part of the Brillouin zone in the 
fermion loop integrals:

$$
\begin{aligned}
\Theta_{m n} & =\frac{1}{V} \sum_{p \in \mathcal{B}} \exp [i p(m-n) a] \Theta(p) \\
& =\frac{1}{N^{D}} \prod_{\mu} \frac{\sin \left[\pi\left(m_{\mu}-n_{\nu}\right) / 2\right]}{\sin \left[\pi\left(m_{\mu}-n_{\nu}\right) / N\right]},
\end{aligned}
$$

where $\Theta(p)$ is equal to unity if $p_{\mu} \in \mathcal{D}(\bmod 2 \pi)$, or to zero otherwise. $V$ is the volume and $N^{D}=$ $V / a^{D}$ is the number of sites of the $D$-dimensional lattice; when $V \rightarrow \infty$, the sum $(1 / V) \sum_{p \in \mathcal{D}} \rightarrow$ $\int_{\mathcal{D}} d^{D} p /(2 \pi)^{D}$.

Because of the presence of $\Theta$ under the trace sign, the effective action now cannot be written in terms of the determinant of some operator. It, however, has the following constructive representation:

$$
\begin{aligned}
\operatorname{Tr} \Theta \ln [ & \left.\nabla(U) \nabla^{-1}(1)\right]=\int_{0}^{1} d t \operatorname{Tr}\{\nabla(U-1) \\
& \left.\times \Theta[\nabla(1)+t \nabla(U-1)]^{-1}\right\},
\end{aligned}
$$

where $t \in[0,1]$ is a real parameter. Note, that though $\Theta$ is non-local, it brings no much problems, since it is not gauged, and the main price is actually the integration over $t$.

3. Sketch of basic properties of the ansatz. The contribution to $\Gamma[A]$ of the $n$th order in $g A$ has the form

$$
\begin{gathered}
\Gamma_{n}[A]=\int_{\mathcal{B}} \frac{d^{D} q_{1}}{(2 \pi)^{D}} \cdots \frac{d^{D} q_{n}}{(2 \pi)^{D}} \delta\left(q_{1}+\cdots+q_{n}\right) \\
\times \operatorname{tr}\left[g A_{\mu_{1}}\left(q_{1}\right) \cdots g A_{\mu_{n}}\left(q_{n}\right) \Gamma_{\mu_{1} \cdots \mu_{n}}(q)\right] \\
\Gamma_{\mu_{1} \cdots \mu_{n}}(q)=\int_{\mathcal{D}} \frac{d^{D} p}{(2 \pi)^{D}} \Gamma_{\mu_{1} \cdots \mu_{n}}(q ; p),
\end{gathered}
$$

where $p$ is the fermion loop momentum. Due to the global chiral invariance each $\Gamma_{n}[A]$ is decomposed into a sum of two terms differing from each other only in the presence or absence of $\gamma_{5}$ in $\Gamma_{\mu_{1} \cdots \mu_{n}}(q ; p)$. The terms without $\gamma_{5}$ contributes to $\Re \Gamma$. The terms with $\gamma_{5}$ contributes to $\Im \Gamma$. From the properties of the traces of $\gamma$-matrices it follows that the real part of $\operatorname{tr} \Gamma_{\mu_{1} \cdots \mu_{n}}(q ; p)$ is periodic, and its imaginary parts is antiperiodic functions of $p$ in the domain $\mathcal{D}$.

These features of the functions $\operatorname{tr} \Gamma_{\mu_{1} \ldots \mu_{n}}(q ; p)$ determine the main properties of the ansatz. In particular, from here it follows that our ansatz yields a gauge invariant result for $\Re \Gamma[A]$, which is

$\Re \Gamma[A]=\frac{1}{2^{D}} \Gamma_{\text {naive }}[A]=\frac{1}{2^{D / 2}} \Re \Gamma_{\text {staggered }}[A]$,

and that the gauge non-invariance of $\Im \Gamma[A]$ has a simple origin very similar to what one has in the continuum theory. Indeed, making use of Ward's identities, the expression for gauge variation of $\Gamma_{n}[A], \delta \Gamma_{n}[\omega, A]$, can be presented in terms of the differences of two momentum integrals over the domain $\mathcal{D}$ with the integrands differing from each other by a shift of the loop momentum. Due to periodicity of the real parts of the integrands in the domain $\mathcal{D}$ one can make appropriate shifts of the integration variable that results in $\delta \Re \Gamma_{n}[\omega, A]=0$ for any $n$. For the imaginary parts $\mathcal{D}$ is not the period of the corresponding integrands and such shifts result in the appearance of a kind of surface terms, which vanish when the regulator is removed, provided the shifts are finite and the corresponding integrals converge or at most diverge logarithmically. For infinitesimal gauge transformation $\omega$ the quantity $\delta \Im \Gamma_{n}[\omega, A]$ has the form

$$
\begin{aligned}
\delta \Im \Gamma_{n}[\omega, A] & =\int_{\mathcal{B}} \frac{d^{D} q_{1}}{(2 \pi)^{D}} \cdots \frac{d^{D} q_{n-1}}{(2 \pi)^{D}} \operatorname{tr}\left[g \omega \left(-q_{1}\right.\right. \\
& \left.\left.-\cdots-q_{n-1}\right) g A_{\mu_{1}}\left(q_{1}\right) \cdots g A_{\mu_{n-1}}\left(q_{n-1}\right)\right] \\
& \times \delta \Im \Gamma_{\mu_{1} \cdots \mu_{n-1}}(q),
\end{aligned}
$$

and simple estimates show that

$$
\delta \Im \Gamma_{\mu_{1} \cdots \mu_{n-1}}(q)=O\left(a^{n-D}\left(q_{1}+\cdots+q_{n-1}\right)\right) .
$$

Hence, for smooth external fields $A$, i.e. such $A$, that $\lim _{a \rightarrow 0} A_{\mu}(q)=0$ for any finite $q a$, all the terms $\Im \Gamma_{n>D}[A]$ are gauge invariant in the continuum limit, while the terms $\Im \Gamma_{n \leq D}[A]$ give rise to the anomalies.

In [1] it was shown that the ansatz reproduces correct continuum limit for convergent contributions to $\Gamma[A]$ of any finite order in smooth $A$, as well as consistent chiral anomalies. Thus for smooth external fields our ansatz is almost perfect.

4. The problem arises for non-smooth external fields, i.e. when the external momenta $q$ are no longer kept finite when the lattice spacing tends to zero. Such a situation is realized in 
the full theory, where the integration is performed over the gauge degrees of freedom as well. As it is seen from (9), at $q=O(1 / a)$ the gauge variation of the contribution of the $D+1$ th order to the effective action is no longer suppressed by a power of $a$; the feature of the effective action that was a mild imperfection for smooth $A$ causes the serious problem for non-smooth $A$. In our formulation the problem is soften by the fact that due to presence of fermion modes of the opposite chirality at high momenta [1], the imaginary part of $\Gamma(q)$ in (6) vanishes near the boundary of the Brillouin zone. However, due to the properties of the trigonometric functions we expect that $\Im \Gamma(q)$ drop sufficiently fast at $q>f / a$, where $f$ is some fraction of $\pi$. So the region $q \leq O(f / a)$ may be still dangerous.

The estimation (9) of the gauge variation of $\Gamma[A]$ is based on the power counting arguments and, therefore, is quite general. Thus, we conclude that this problem is common to all imperfect formulations of the chiral gauge theories, including, in particular, the overlap formula [2] (see also [7] for another evidence).

5. Need for another scale. A universal way to suppress this gauge non-invariance is to limit the domain of changing of the external momenta $q$ to a region determined by a new scale $b \gg a$, such that $\lim _{b \rightarrow 0} a / b=0$. So, now $q \leq O(1 / b)$ and in the absence of anomalies the gauge noninvariance of $\Im \Gamma[A]$ is controlled by the ratio $r=$ $a / b \ll 1$. The question is how much this control is effective on a finite lattice.

We can get some idea of that applying expressions (8) and (9) to the generic case $g A_{\mu}(n+$ $\hat{\mu} / 2)=O(1 / b)$ and $g \omega(n)=O(1)$. Returning to the finite lattice, we find

$\delta \Im \Gamma_{n}[\omega, A]=O\left(N_{b}^{D} r^{n-D}\right)$.

where $N_{b}^{D}=V / b^{D}$. Although this estimate is formall, it indicates that the achieving the gauge invariance in generic case may be more difficult than that in the perturbative regime, and that the infinite volume limit should be correlated with the

\footnotetext{
2 Eqs. (8), (9) are based on the expansion of the effective action in powers of $g A$, that implies $\left|g A_{\mu}(n+\hat{\mu} / 2)\right| \ll$ $1 / b$ and $g \omega(n) \ll 1$ and corresponds to the perturbative regime.
}

limit $r \rightarrow 0$.

Thus, even mild imperfection of the effective action leads to the necessity for introducing a new scale to the gauge sector. This can be done either by imposing constraints on the gauge variable measure (see [6] and references therein) or by defining the gauge variables on the sublattice with the spacing $b$, 5 , with their subsequent interpolation to the original lattice (then $N_{b}^{D}$ in (10) is the number of sites of such a sublattice). So, in this case at least part of the problems of the chiral gauge theories moves to the gauge sector.

To conclude, we note that the only known exactly gauge invariant formulation of the lattice chiral gauge theory 8 (employing non-local SLAC derivative) also involves the additional scales, which in this case are (generalized) PauliVillars masses. Some arguments for the insufficiency of a single scale lattice are given as well by the random lattice approach [9]. So, it appears to be plausible that a general no-go statement about impossibility to formulate chiral gauge theory on a lattice with only one scale is hold.

I am grateful to the International Science Foundation and Organizing Committee of the "Lattice '96" for financial support, and to Y. Shamir and A. A. Slavnov for interesting discussions. This work was partly supported by the Russian Basic Research Fund under the grant No. 95-02-03868a.

\section{REFERENCES}

1. S. V. Zenkin, hep-lat/9603014.

2. R. Narayanan and H. Neuberger, Nucl. Phys. B 433 (1995) 305; R. Narayanan, these proceedings.

3. G. T. Bodwin and E. V. Kovács, Nucl. Phys. B (Proc. Suppl.) 30 (1993) 617.

4. P. Hernandez and R. Sundrum, Nucl. Phys. B 455 (1995) 287; R. Sundrum, these proceedings.

5. G. T. Bodwin, hep-lat/9510002; these proceedings.

6. Y. Shamir, hep-lat/9509023.

7. Y. Kikukawa, these proceedings.

8. A. A. Slavnov, Phys. Lett. B 348 (1995) 553.

9. T. W. Chiu, these proceedings. 